\title{
2D Simulations of Supernovae
}

\author{
By DAVID ARNETT
}

Steward Observatory, University of Arizona, Tucson, AZ 85721, USA

\section{Introduction}

Several multidimensional computations of hydrodynamics related to supernovae have been completed, and are summarized here. More detail may be found in Arnett 1994a,b, Arnett \& Livne 1994a,b, and Livne \& Arnett 1993. The hydro code PROMETHEUS is based upon an implementation of the piecewise-parabolic method (PPM) of Colella \& Woodward 1984, as described in Fryxell et al. 1991. A detailed comparison of PPM with other schemes is given in Woodward \& Colella 1984. The method constructs the physics of the flow between grid points by a nonlinear solution of the equations of continuity of mass, momentum and energy (the Riemann problem) rather than the usual mathematical approach of a Taylor expansion about the grid points. This gives it better resolution per grid point, which is highly desirable for multidimensional problems. Although the effort required per grid point is greater, the number of such points is less (often much less) for a given level of accuracy. Because the computational load per grid point is greater, more realistic physics (reactions, radiation, gravity, etc.) may be added before affecting the runtime significantly. Thus PPM is well suited for multidimensional problems with significant physics beyond the bare hydrodynamics.

The Prometheus project was an effort by the author, Bruce Fryxell and Ewald Müller, to implement a "state of the art" hydrodynamic method with realistic microphysics for stellar problems. After an extensive study of several methods, and direct comparison of the resulting codes (Fryxell, Müller \& Arnett 1989), PPM was chosen as the preferred method. The first version of the PROMETHEUS code was written by Fryxell and Müller. It is an extension of basic PPM in that (1) it includes an arbitrary number of separate fluids to keep track of the abundance of different nuclear species, (2) nuclear reactions are included (see below), and (3) modifications were made to allow use of a realistic (not a gamma law) equation of state (Colella \& Glaz 1985). Variations of this original code have been used by several other groups (e.g., Blondin, et al. 1991, Cattaneo, et al. 1991, and Burrows, this volume) as well as various collaborations involving Fryxell, Müller and Arnett. PROMETHEUS has a number of added features which are crucial for many scientific problems. It allows the user to choose a coordinate system: 1, 2, and 3 dimensions, with cartesian, cylindrical or spherical coordinates. It has the option of a moving grid, with the grid motion to be defined as needed. This gives enormous improvement in resolution for many stellar problems. Fryxell wrote a superb graphics interface; the slides resulting from it have been widely used by our group and others. PROMETHEUS is a highly structured code, and routinely benchmarks well on vector and modestly parallel computers.

An extensive review (Fryxell, Müller \& Arnett 1989) of our first experiences with the coupling of a reaction network (an alpha chain to ${ }^{56} \mathrm{Ni}$ ) was widely distributed in preprint form, and was to be published in 1989, but has not appeared (due to no fault of our own). Although the work described below relating to reaction networks is new in this context, it used insights gained in the collaborative effort, and represents an extension 
of PROMETHEUS to deal with a new set of problems. Video movies of the following four studies were shown.

\section{Supernovae of Type II}

\subsection{Static Oxygen Shells}

With new hydrodynamic techniques, the relatively fast evolutionary stages of a star prior to core collapse may be explicitly computed in two spatial dimensions, with a treatment of the microphysics (e.g., nuclear reactions, equation of state, neutrino cooling) which is comparable to typical one-dimensional simulations. The nature of shell oxygen burning in a massive star, prior to core collapse, is used as first example; it is of particular interest because it is (1) the region in which ${ }^{56} \mathrm{Ni}$ will be produced by the supernova shock, (2) the region of the "mass cut", which will separate the collapsed core from the ejected mantle, (3) the site of much of the explosive nucleosynthesis, and (4) a suggested source of symmetry breaking to drive mixing instabilites which were observed in SN1987A. The nature of the shell burning affects the size of the core which will collapse. The method is illustrated on a test case, and the character of the convection is examined. The initial model contained the oxygen burning shell and the overlying convective region, from a star of $20 M_{\odot}$ (a model for the progenitor of SN1987A). It is representative of stars of this mass range, as well as helium cores of about $6 M_{\odot}$. For efficiency, the computational domain was a wedge of about 30 degrees.

Figure 1 shows contours of the ${ }^{12} \mathrm{C}$ abundance, which is a sensitive (inert) tracer of exposure to the oxygen burning flame zone, after 145 seconds. The spherical symmetry is broken; this time is already about $1 \%$ of the evolutionary time for such shell burning.

The first conclusion is that multidimensional hydrodynamics methods have matured to the point that they can provide new insights into difficult theoretical problems in stellar astrophysics. These preliminary computations can be easily surpassed with more powerful technology (by simply switching from a workstation to a supercomputer, or using the next generation workstation), and by more sophisticated use of existing algorithms. In particular, since these simulations were completed, efforts on another topic led to the development of an algorithm for semi-lagrangian grid motion, in which mass flow out of the boundaries is much less than seen here. That will provide a more natural tool for such stellar problems, and should allow evolution to be extended to significantly longer times. These simulations demonstrate that oxygen burning shells do approach a thermal steady state, as has been assumed for many years (Arnett 1972a). They show that the profile in abundance of ${ }^{16} \mathrm{O}$ fuel does wander away from the steep form that one dimensional stellar evolutionary codes tend to give, and give a plausible quantitative estimate of this effect. Further, a new view of the structure of the burning and convective shell appears, in which the density (buoyancy) perturbations and the convective flows interact because of the burning. This is a consequence of the rapidity of the heating, relative to the milder situation confronted in earlier stages of stellar evolution. However, no light is yet shed on exactly how burning shells move out in mass (Arnett 1972b), which remains a key uncertainty for quantitative estimates of nucleosynthetic yields and massive star structure. On the contrary, these results strengthen the worry that important aspects of the physics of the late evolution of massive stars are still not sufficiently well understood. An understanding of this longer term, "secular" evolution of the oxygen burning shell still eludes us, but with these new tools, it may now be within our capability.

The convective motions simulated here are only two dimensional, have a changing (not static) background state, and are strongly coupled to the energy release by nuclear 


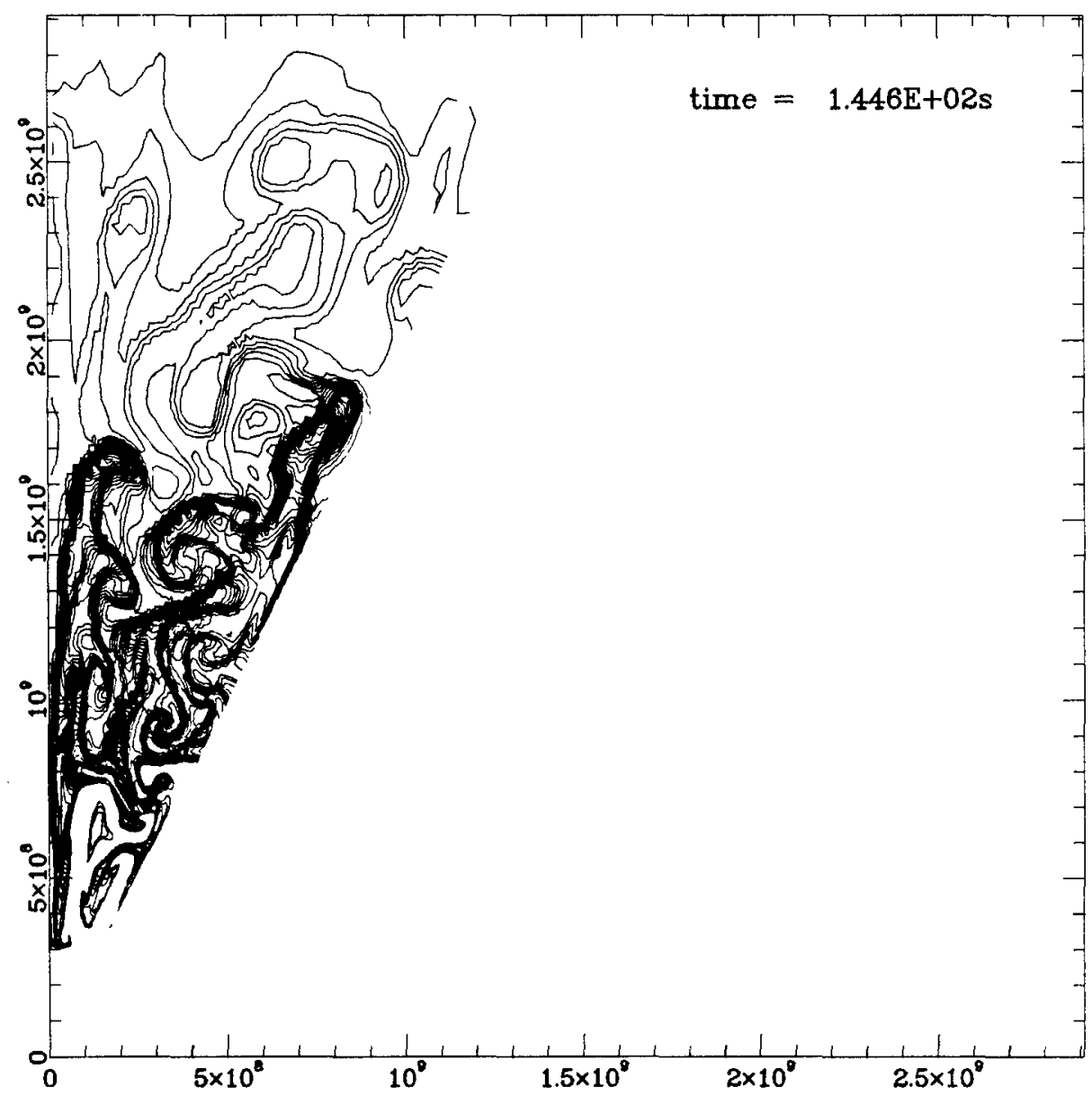

Figure 1. Contours of ${ }^{12} \mathrm{C}$ abundance.

burning. Even so, there are similarities to the three dimensional, turbulent convection simulated by Cattaneo, et al. 1991, which was driven only by a simple temperature gradient. For example, the distinction between strong, coherent long lived motions and weaker, disorganized ones is valid here as well; the former dominate the transport of composition, while the latter seem more closely related to net energy flow. Although demanding computationally, an extension of the present efforts into three dimensions and to longer times would provide the basis for a direct comparison.

These simulations give a definite prediction for the amplitude of nonspherical perturbations present in a presupernova. In the oxygen shell, fractional density perturbations of $2.5 \%$ and velocity perturbations of about $0.8 \%$ of sonic will occur. This sets a lower limit, because as the core burns silicon and then contracts toward collapse, the oxygen shell will burn more vigorously (Arnett 1977). Such values are similar to those used in Arnett, Fryxell \& Müller 1989, Müller et al. 1991, and Fryxell et al. 1991.

\subsection{Dynamic Oxygen Shells}

The evolution of an oxygen burning shell is simulated from the beginning of core collapse through explosive nucleosynthesis. Again, the hydrodynamic behavior is computed in two 
spatial dimensions, with a treatment of the microphysics (e.g., nuclear reactions, equation of state, neutrino cooling) which is comparable to typical one-dimensional simulations. The initial parameters are taken from the previous simulation, in two dimensions, of stationary shell oxygen burning in a massive star prior to core collapse. Previous one dimensional computations are used to define the boundary conditions for the infall and the explosion stages. The simulations produce estimates for (1) the ${ }^{56} \mathrm{Ni}$ produced by the supernova shock, (2) the "mass cut", which separates the collapsed core from the ejected mantle, (3) the explosive nucleosynthesis, and (4) symmetry breaking to drive mixing instabilites which were observed in SN1987A.

After silicon burning increases the mass of the iron core in a massive star, core collapse begins (e.g., Arnett 1977). Initially the contraction is slow, with first the silicon and then the oxygen shells burning vigorously as they move to smaller radii. A second stage follows: as the core contraction accelerates into collapse, an explosion occurs (the details of which are actively debated at present), and a shock propagates outward, heating and ejecting the oxygen burning layer. Here we focus not on the explosion mechanism, but on its implications for the oxygen burning shell.

During the later stages of oxygen burning, electron capture increases the neutron excess so that ${ }^{56} \mathrm{Ni}$ is no longer the end product of nuclear burning. The iron-peak nuclei which are formed (mostly ${ }^{54} \mathrm{Fe}$ and ${ }^{58} \mathrm{Ni}$ ) have a much lower cosmic abundance than the ${ }^{56} \mathrm{Fe}$ to which ${ }^{56} \mathrm{Ni}$ decays via the intermediate ${ }^{56} \mathrm{Co}$. The observed abundances in solar system matter suggest that (usually) this matter does not escape the star. Thus two important questions, the production of ${ }^{56} \mathrm{Ni}$ to power the light curve and the size of the condensed remnant, are related to what happens in this stage of the evolution of the star.

The mass of ${ }^{56} \mathrm{Ni}$ ejected ranged from 0.03 to $0.20 M_{\odot}$ for explosive energies of 1 to 2 foe. The nucleosynthesis conditions were similar to those needed for production of solar system abundances. The amount of mass which falls back onto the neutron star is sensitive to the inner boundary condition, i. e., to the explosion mechanism. Figure 2 shows a contour plot of the abundance of ${ }^{56} \mathrm{Ni}$ after shock passage. The entropy generated by explosive oxygen burning drives vorticity production and amplifies the nonspherical perturbations left from the previous convective stage (see above).

\section{Supernovae of Type Ia}

\subsection{Deflagration}

The nature of the "delayed detonation" mechanism (Khokhlov 1991) for the explosion of Type Ia supernovae is investigated by use of two-dimensional numerical hydrodynamics simulations. A new algorithm is used to treat the deflagration front (Arnett \& Livne 1994a); it forces the burning rate to be that implied by the speed of the front. Assuming that the front propagates locally at the laminar flame speed (Timmes \& Woosley 1992), the deflagration is insufficient to unbind the star. Expansion shuts off the flame; much of this small production of iron group nuclei occurs at lower densities, which reduces the electron-capture problem. The burning front does become wrinkled, but the wavelength of the instability is much larger than the computational grid size, and is resolved (see Livne, this volume); this is consistent with previous analysis (Livne \& Arnett 1993).

Because the degenerate star has an adiabatic exponent only slightly above $\frac{4}{3}$, the energy released by deflagration drives a pulsation of large amplitude (see Khokhlov 1991 and also Nomoto, et al. 1976). During the first expansion phase, adiabatic cooling shuts off the burning, and a Rayleigh-Taylor instability then gives mixing of high entropy ashes with low entropy fuel. During the first contraction phase, compressional heating re-ignites 


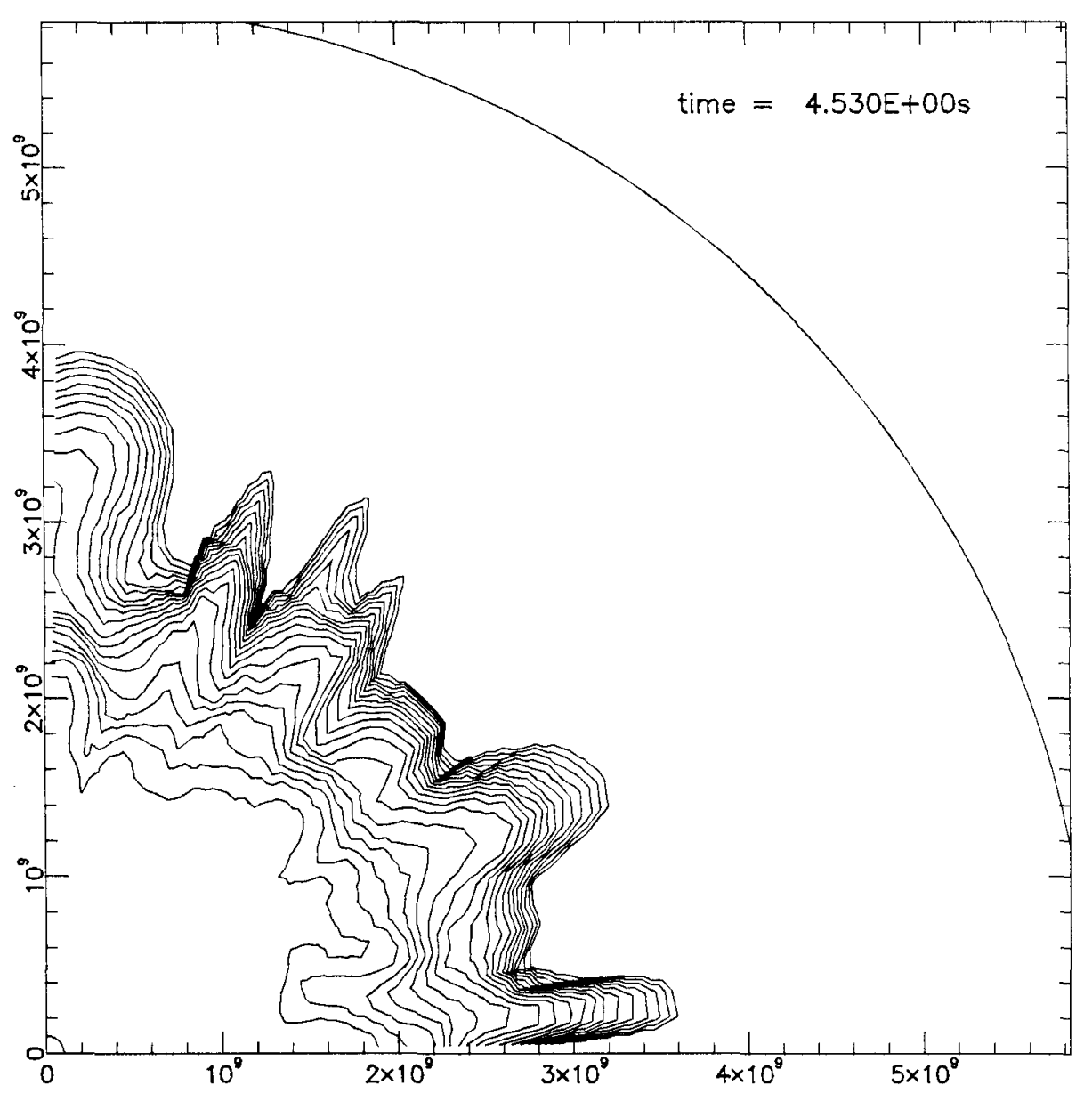

Figure 2. Contours of ${ }^{56} \mathrm{Ni}$ abundance.

the material. This section deals with the deflagration phase, from the onset of burning, through expansion and quenching of the flame, to the first contraction.

Figure 3 shows contours of the heating rate from the deflagration front, after $0.21 \mathrm{~s}$. The instability of the front is obvious. As time passes, the smaller "ripples" merge with larger ones, giving fewer but larger perturbations.

The new method for treating stellar deflagrations with complex nucleosynthesis seems successful, and works well with our PPM code. It is computationally efficient, and produces results which are consistent with mathematical analysis of similar systems (e.g., Zeldovich, et al. 1985), and with previous numerical results obtained with very different methods (Livne 1993).

Given the assumption that the propagation velocity is given by that computed for laminar flow, no explosion results during the deflagration phase. This is in contradiction to the standard "deflagration model" scenario which is widely used to explain Type Ia supernovae (e.g., Nomoto, et al. 1984, Woosley 1990).

The deflagration front is unstable toward wrinkling, as Figure 3 illustrates, but the larger wavelengths grow at the expense of the smaller ones. This is in contradiction with what is generally assumed in the astronomical literature (see Woosley 1990). 
96

David Arnett: 2D Simulations of Supernovae

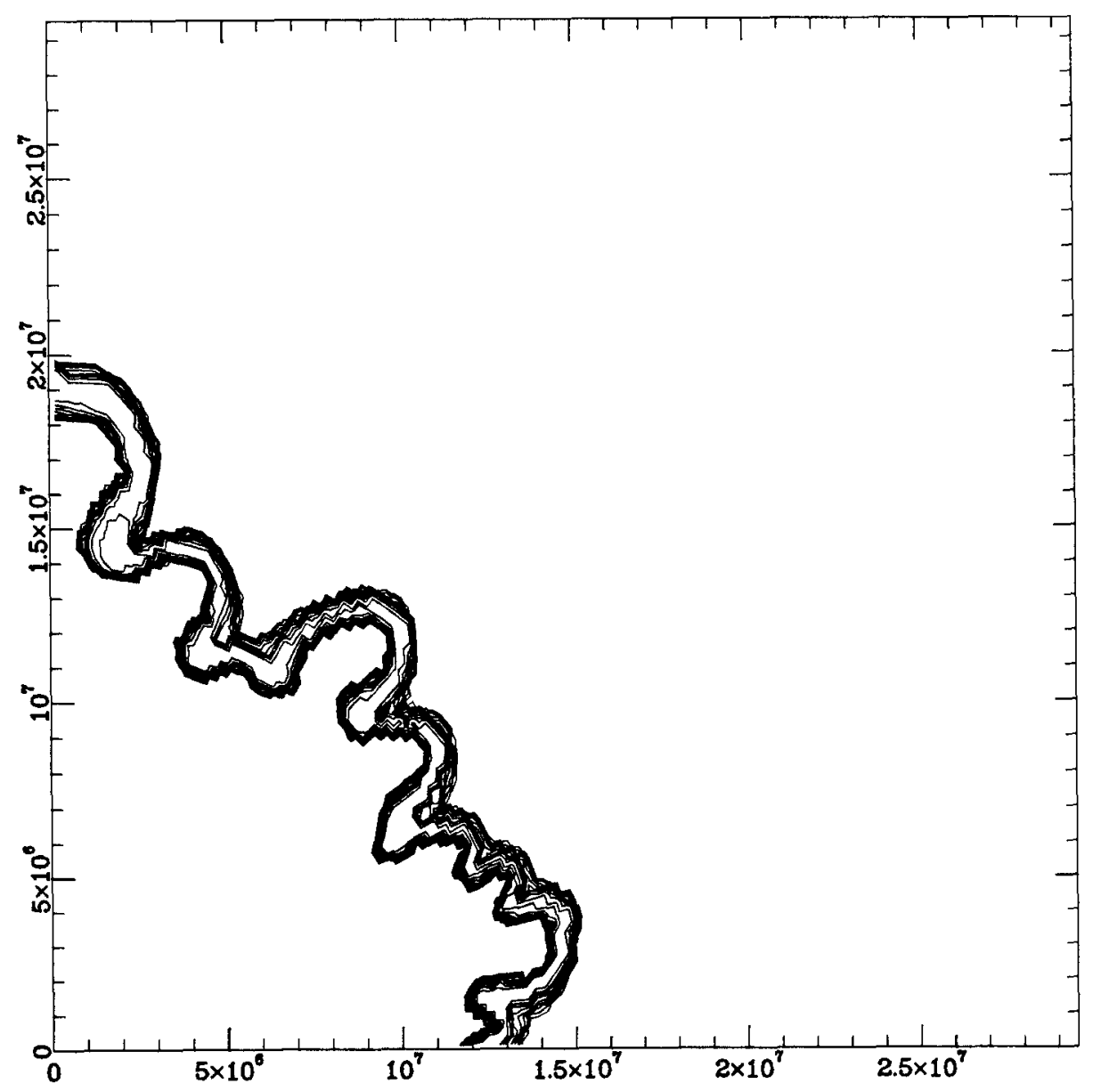

Figure 3. Contours of nuclear heating rate in deflagration front.

The amount of neutron enrichment due to electron capture on free protons and on ${ }^{56} \mathrm{Ni}$ is sufficiently small that moderate amounts of ${ }^{56} \mathrm{Ni}$ (about $0.1 M_{\odot}$ ) can dilute the mixture to solar proportions. The problem of excessive neutronization is eased by a consequence of the two dimensional aspect of the hydrodynamic motion: the newly burned matter rises and expands to lower density, which slows electron capture. Two dimensional computations also give more burning than predicted by spherical calculations; the front wrinkles, and has a larger area, which gives a faster net consuption of fuel.

The deflagration reduces the gravitational binding of the star to about half its initial value. This drives an overall expansion. Because the adiabatic exponent is close to $\frac{4}{3}$, the amplitude of this expansion is large. This reduces the burning rates to negligible values, and allows a mixing of the hot ashes and cold fuel. Upon contraction, this matter is compressionally heated to temperatures at which burning becomes vigorous again.

3.2. Detonation

The investigation, by use of two-dimensional numerical hydrodymanics simulations, of the "delayed detonation" mechanism (Khokhlov 1991) for the explosion of Type Ia sipernovae was continued. During the first expansion phase, adiabatic cooling shuts off the

https://doi.org/10.1017/S0252921100007958 Published online by Cambridge University Press 
burning, and a Rayleigh-Taylor instability then gives mixing of high entropy ashes with low entropy fuel. During the first contraction phase, compressional heating re-ignites the material. The burning was allowed to develop into a detonation using the nonspherically symmetric models. Although it begins from several "hot spots", the detonation grows toward spherical symmetry at late times. At these densities $\left(\rho \sim 10^{7}\right.$ to $\left.10^{8} \mathrm{~g} \mathrm{~cm}^{-3}\right)$, ${ }^{56} \mathrm{Ni}$ and nuclei of the Si-Ca group are the dominant products of the burning. The bulk yields are sensitive to the density of the star when the transition to detonation occurs.

These preliminary computations represent a self-consistent treatment from onset of deflagration to after the explosion. Bulk yields and velocity structure for two-dimensional models are now available. The crucial theoretical question which arises is the density at which transition to detonation occurs. The only models which give velocities higher than $8,000 \mathrm{~km} \mathrm{~s}^{-1}$, as suggested by observations of SNIa spectral lines, do so by burning the underlying matter to ${ }^{56} \mathrm{Ni}$. This implies, that if these promising models are in fact correct for SNIa's, then the minimum amount of ${ }^{56} \mathrm{Ni}$ produced is $0.7 M_{\odot}$. This implies a relatively high intrinsic brightnerss at maximum, and argues against the smaller values of the cosmic distance scale. Crudely speaking, Hubble constants above $80 \mathrm{~km} \mathrm{~s}^{-1} \mathrm{Mpc}^{-1}$ are unlikely. More to the point, refined use of this effect may give an accurate estimate of the minimum absolute brightness of SNIa's, as well as observational opportunities for internal tests of the procedure.

\section{Acknowledgements}

I wish to thank Eli Livne and Alexei Khokhlov for many useful disussions.

\section{REFERENCES}

Arnett, D. 1972a, Ap. J., 173, 393

Arnett, D. 1972b, Ap. J., 176, 699

Arnett, D. 1977, Ap. J., 218, 815

Arnett, D. 1994a, Ap. J., 427, 932

Arnett, D. 1994b, in preparation.

Arnett, D., Fryxell, B. A., \& Müller, E., 1989, Ap. J. Lett., 341, L63

Arnett, D. \& Livne, E., 1994a, Ap. J., 427, 315

Arnett, D. \& Livne, E., 1994b, Ap. J., 427, 320

Blondin, J. M., Stevens, I. R., \& Kallman, T. R., 1991, Ap. J., 371, 684

Cattaneo, F., Brummel, N. H., Toomre, J., Malagoli, A., \& Hurlburt, N. E., 1991, Ap. J., 370, 282

Colella, P. \& Glaz, H. M., 1985, J. Comput. Phys., 59, 264

Colella, P. \& Woodward, P. R., 1984, J. Comput. Phys., 54, 174

Fryxell, B. A., Müller, E. \& Arnett, D., 1989, Numerical Methods in Astrophysics, ed. Paul R. Woodward, to have been published in Computational Techniques by Academic Press; preprint MPA 449, Max-Planck-Institut für Astrophysik, Garching

Fryxell, B. A., Müller, E. \& Arnett, D., 1991, Ap. J., 367, 619

Khokhlov, A. 1991, A. \& Ap., 245, L25

Livne, E., 1993, Ap. J. Lett., 406, L17

Livne, E. and Arnett, D., 1993, Ap. J. Lett., 415, L107

Müller, E., Fryxell, B. A., and Arnett, W. D., 1991, A. \& Ap., 251, 505

Nomoto, K., Sugimoto, D., and Neo, S. 1976, Astrophys. Space Sci., 39, 137

Nomoto, K., Thielemann, F. K., \& Yokoi, K. 1984, Ap. J.286, 644 
Timmes, X. and Woosley, S. E., 1992, Ap. J., 396, 649

Woodward, P. R., \& Colella, P. 1984, J. Comput. Phys., 54, 115

Woosley, S. E., 1990, in Supernovae, ed. A. G. Petschek (Dordrecht: Kluwer), 182.

Zeldovich, Ya. B. Barenblat, G. I., Librovich, V. B., \& Mikhviladze, G. M., 1985, (New York: Consultants Bureau/Plenum). 